\title{
Focusing the neuroscience and societal implications of cognitive enhancers
}

George Savulich ${ }^{1,2}$, Thomas Piercy ${ }^{1}$, Annette B. Bruhl ${ }^{1,2,3}$, Chris Fox ${ }^{4}$, John Suckling ${ }^{1,2}$, James B. Rowe ${ }^{2,5,6}$, John T. O'Brien ${ }^{1}$ and Barbara J. Sahakian ${ }^{1,2^{*}}$

1. Department of Psychiatry, University of Cambridge, Cambridge, UK

2. Behavioural and Clinical Neuroscience Institute, University of Cambridge, Cambridge, UK

3. Department of Psychiatry, Psychotherapy and Psychosomatics, Psychiatric Hospital, University of Zurich, Zurich, Switzerland

4. Department of Psychological Sciences, Norwich Medical School, University of East Anglia, Norwich, UK

5. Department of Clinical Neurosciences, University of Cambridge, Cambridge, UK

6. MRC Cognition and Brain Sciences Unit, University of Cambridge, Cambridge, UK

*Corresponding author: Professor Barbara J. Sahakian, Department of Psychiatry, University of Cambridge, Herchel Smith Building for Brain and Mind Sciences, Forvie Site, Robinson Way, CB2 0SZ, Cambridge, UK; bjs1001@medschl.cam.ac.uk

Keywords: cognitive enhancement, 'smart drugs', cognitive training using games, nootropics, ethics, society

Number of Tables: 0

Number of Figures: 1

Words: Introduction: 73 (max: 75); Main body: 1,500 (1,500 max)

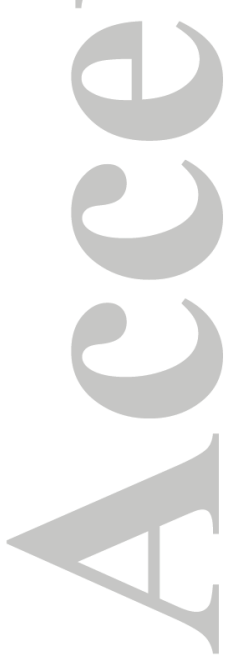

This article has been accepted for publication and undergone full peer review but has not been through the copyediting, typesetting, pagination and proofreading process which may lead to differences between this version and the Version of Record. Please cite this article as an 'Accepted Article', doi: 10.1002/cpt.457 


\section{Introduction}

Cognitive enhancement can benefit the individual and society, but also has associated risks and ethical concerns. Cognitive-enhancing drugs are used in the treatment of neuropsychiatric disorders. Non-pharmacological strategies are also emerging, which have the potential to improve motivational deficits associated with neuropsychiatric symptoms and should be prioritized for development. The increasing lifestyle use of 'smart' and other drugs indicates the desire for healthy people to improve themselves. Safety and ethical implications are discussed.

\section{Restoring cognitive function}

Cognitive dysfunction is characteristic of neuropsychiatric disorders, creating a clear need for effective strategies to restore, for example, attentional biases, aberrant learning, dysfunctional reward systems and dysregulation in top-down cognitive control by the prefrontal cortex ${ }^{1}$.

Disturbances in emotional and motivational processes, such as apathy and withdrawal, affect outcome and remain complex barriers to treatment entry and engagement. These challenges impact on the functionality and wellbeing of patients and their carers. Each year, the direct and indirect costs of neuropsychiatric disorders are widespread, with implications for the government (increasing demands on healthcare/social services), the economy (lost productivity/earnings) and the quality of patient life (difficulty to live/work independently).

While cholinesterase inhibitors, such as donepezil, and stimulants, such as methylphenidate, are used in the treatment of cognitive symptoms in Alzheimer's disease and attention deficit hyperactivity disorder (ADHD) respectively, there are as yet no drugs approved by the

FDA/EMA/MHRA for the treatment of cognitive dysfunction in depression, schizophrenia or mild cognitive impairment (MCI). Improving cognitive dysfunction could help alleviate some of the economic and societal costs of disease burden. Cognitive-enhancing drugs would also 
be useful for disorders in which cognitive impairments persist even after successful treatment of the more acute symptoms (e.g. low mood in depression, psychotic symptoms). In addition to drugs, non-pharmacological cognitive-enhancers can be used to treat cognitive dysfunction in neuropsychiatric and neurodegenerative disorders. These include brain stimulation devices and cognitive training packages that are designed to improve neural circuitry leading to neuroplasticity. The clearest benefits found thus far are in depression (improvement in treatment-resistant symptoms following transcranial magnetic stimulation) and schizophrenia (cognitive remediation of memory deficits) $)^{1,2}$.

Computer games are highly rewarding and, in our opinion, an exciting option for delivering targeted cognitive training. We previously demonstrated that gamified cognitive training (www.peak.net/advanced-training; Figure 1) can improve episodic memory and functional outcome in patients with schizophrenia ${ }^{1}$. Importantly, high levels of enjoyment and desire to continue were maintained throughout all hours of gameplay, which we believe represents a motivational improvement over traditional (usually boring) cognitive training paradigms.

Cognitive training is also effective for improving cognition and functional abilities in individuals with $\mathrm{MCI}^{3}$. Pharmacological interventions that slow progression from MCI to Alzheimer's disease are currently under development. As cholinesterase inhibitors are most likely to be effective in the treatment of attentional dysfunction, alternative strategies for treating memory problems are needed. Despite the potential benefits of cognitive training, the cost and inconvenience of delivery (e.g. specialized equipment, participant travel, supervision), in addition to motivational deficits associated with apathetic or depressive symptoms often characteristic of older populations with memory difficulties, leads to considerable dropout. We propose that gamified cognitive training could circumvent dropout rates by maximizing engagement in patients with deficits in motivation, primarily in MCI but 
also ADHD, and further extend to other populations with a high prevalence of neuropsychiatric symptoms, such as traumatic brain injury.

Gamified cognitive training studies with longitudinal designs, active control groups and measures of brain and behavioral changes will be critical for evaluating the techniques necessary for cognitive and functional improvement, including the optimal dose and duration of delivery. We further propose that concurrent gamified cognitive training and pharmacological treatments, particularly cognitive-enhancing drugs but also antidepressants and antipsychotics, has potential to improve treatment compliance of patients and synergise the effects needed for good clinical outcome. Combining findings from neuropsychiatry with gaming technology could also reduce some of the stigma associated with mental health treatments.

\section{Enhancing cognitive function}

There is increasing use of cognitive enhancers for 'lifestyle' rather than medical reasons. This is illustrated by modafinil, a wakefulness-promoting agent used in the treatment of sleeprelated disorders. The use of so-called 'smart drugs' by the public indicates the desire (or pressure) for individuals to better their already healthy cognitive functioning.

Work-related stress is the primary reason for healthy individuals using cognitive-enhancing drugs; likewise, students report academic competition and amplified feelings of concentration and wakefulness as their top motivators for smart drug use ${ }^{2}$. Modafinil produces cognitiveenhancing effects in healthy individuals, mostly in higher executive functions such as planning and decision-making. Modafinil also has positive effects on mood. Methylphenidate and amphetamine are two stimulants that improve inhibitory control and memory processes in healthy individuals. Both drugs are classed as Schedule II controlled substances in the USA, 
indicating high abuse potential. Regulation of these drugs is therefore essential but difficult to control, particularly given the sharing and selling of medications following the rise in diagnosis of ADHD in young adults and the ability to purchase medications online.

We recommend physical exercise, education, social interaction, mindfulness and sleep for improving cognitive performance and overall wellbeing. Encouragingly, six months of online cognitive training showed improved cognition, reasoning and activities of daily living in a large number of healthy adults over the age of sixty ${ }^{4}$. Cognitive training of working memory also showed altered functional activation and dopamine receptor density in the brain ${ }^{5}$. It is our opinion that both modafinil and gamified cognitive training can improve task-related motivation, but it may be that gamified cognitive training gives a greater sense of achievement as improvement in performance is attributed to the self, rather than a drug.

The desire to perform better is not limited to students. The term 'psychonauts' describes people who experiment with mind-altering chemicals, sometimes to the extent of taking exact measurements and keeping records of their experiences. Nootropics is the umbrella term that refers to cognitive-enhancing smart drugs, stimulants (prescription medications but also caffeine and nicotine), supplements ('nutracueticals') and other substances such as racetams. Combinations of nootropics called 'stacks' are taken to meet the specific needs of the consumer based on which cognitive function they feel would most benefit from enhancement. Stacks are not FDA-approved for enhancing cognition, but the individual compounds might be approved as dietary supplements. However, the potential harm of mixing nootropics, including short- and long-term side effects, is unknown. In some cases, the quantities of the individual compounds that comprise the final product are not disclosed. Users are therefore turning to online forums to discuss their experiences with nootropics, which, although 
prompting discussion and awareness while maintaining anonymity, could also lead to misrepresentation of their effects and harms.

In parallel to the boom in demand for nootropics, 'microdosing' psychedelics are an increasingly popular phenomenon in which small amounts of psilocybin mushrooms, LSD or mescaline are taken to enhance cognitive function, perception and creativity. This adds a new dimension to what could be considered 'illegal cognitive enhancement'. There is thus an increase in the type of drugs sought for enhancing cognition. The combination of cognitive enhancement with psychoactive effects reflects a change in the use of banned hallucinogenic drugs. Characteristics of users, the long-term effects of microdosing and the extent to which the legal status of psychedelics impacts on their cost and availability pose novel avenues of drug research.

Cognitive enhancement has benefits for jobs that require adaptive learning or attentional shifting under high levels of risk or pressure. Cognitive enhancement could also improve the performance of shift or sleep-deprived workers, thereby increasing safety, work-related efficiency and output. However, a growing number of societal and ethical concerns of smart drug use by healthy people have been raised. Societal concerns are mainly focused on the safety of purchasing prescription medication online from unregulated manufacturing sources. An ethical concern of using smart drugs during exam time, which is regarded by many as cheating, has led some Universities to ban their use. Additional concerns include increased academic pressure and fears of coercion. The lack of randomized controlled trials in healthy people raises a number of health concerns. These include the long-term safety and efficacy of cognitive-enhancing drugs, their potential for misuse and their influence on the developing brain, all of which have not been investigated to an adequate standard. 


\section{Comment}

Reasons for cognitive enhancement are diverse: restoration (treatment), enhancement, workrelated motivation and mild euphoria. We thus call for public-private partnership between the government and pharmaceutical industry to establish the safety and efficacy of currently wellused smart drugs, such as modafinil, in healthy people. Cognitive-enhancing drugs are beneficial for some patients with neuropsychiatric and neurodegenerative disorders, but we also suggest the development of games for cognitive training to simultaneously enhance cognition and motivation. We further call for academia and the hugely successful 'serious' games industry to work together to provide evidence-based non-pharmacological strategies for cognitive enhancement, particularly for disorders in which pharmacological interventions have failed to treat cognitive dysfunction or do not address the associated motivational deficits. We believe that innovation in the field of mental health could improve engagement with non-pharmacological strategies of cognitive-enhancement and alter public perception of treatment through more widespread use. While pharmacological treatments can be of great benefit to patients in terms of cognitive symptoms, other strategies, such as gamified cognitive training, may be beneficial if given in combination with cognitive-enhancing drugs to achieve the best outcome. The advantages of cognitive enhancement should be considered and researched further, but the potential harm for the individual and those around them must also be avoided, to ensure that all members of society flourish. 


\section{References}

1 Sahakian, B.J et al. The impact of neuroscience on society: cognitive enhancement in neuropsychiatric disorders and in healthy people. Philos Trans R Soc Lond B Biol Sci. 370: 20140214 (2015).

2 Brühl, A.B. \& Sahakian, B.J. Drugs, games, and devices for enhancing cognition: implications for work and society. Ann N Y Acad Sci. 1369(1): 195-217 (2016).

3 Li H, Li J, Li N, Li B, Wang P, \& Zhou T. Cognitive training for persons with mild cognitive impairment: A meta-analysis. Ageing Res Rev. 10 285-296 (2011).

4 Corbett, A. et al. The effect of an online cognitive training package in healthy older adults: an online randomized controlled trial. J Am Med Dir Assoc, 16 990-997 (2015).

5 Klingberg, T. Training and plasticity of working memory. Trends Cogn Sci. 14 317-324 (2010).

Funding: GS was funded by grants from Janssen/J\&J and the Wallitt Foundation.

Conflict of interest: Annette Brühl and James Rowe have no competing interests. Chris Fox has received speaker fees from Astellas Pharmaceuticals. John Suckling has acted as a consultant for GlaxoSmithKline. John O'Brien has acted as a consultant for TauRx, Axona and Lilly. Tom Piercy and George Savulich consult for Peak. Barbara Sahakian consults for Cambridge Cognition, Peak, Lundbeck, Servier and Otsuka.

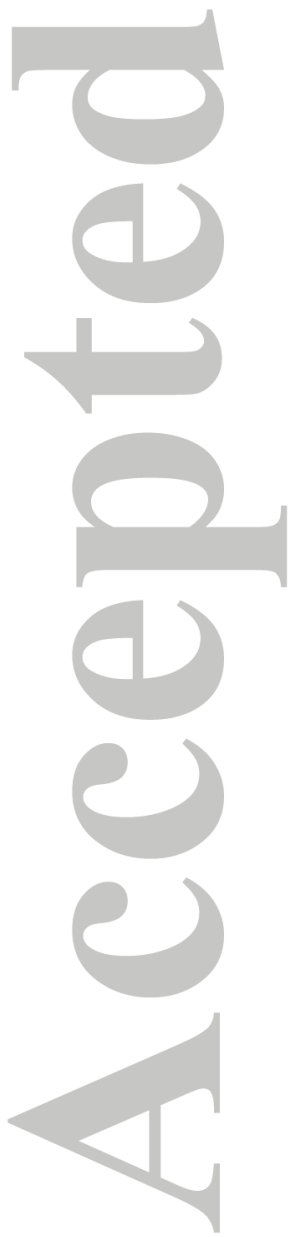


Figure 1. Images from 'Wizard', an example of gamified cognitive training of episodic memory, which embedded a paired associates learning task into a narrative, allowed for the selection of characters, rewarded progress, provided feedback and used visually appealing displays and stimulating music to keep users engaged and motivated (with permission from Peak; www.peak.net)

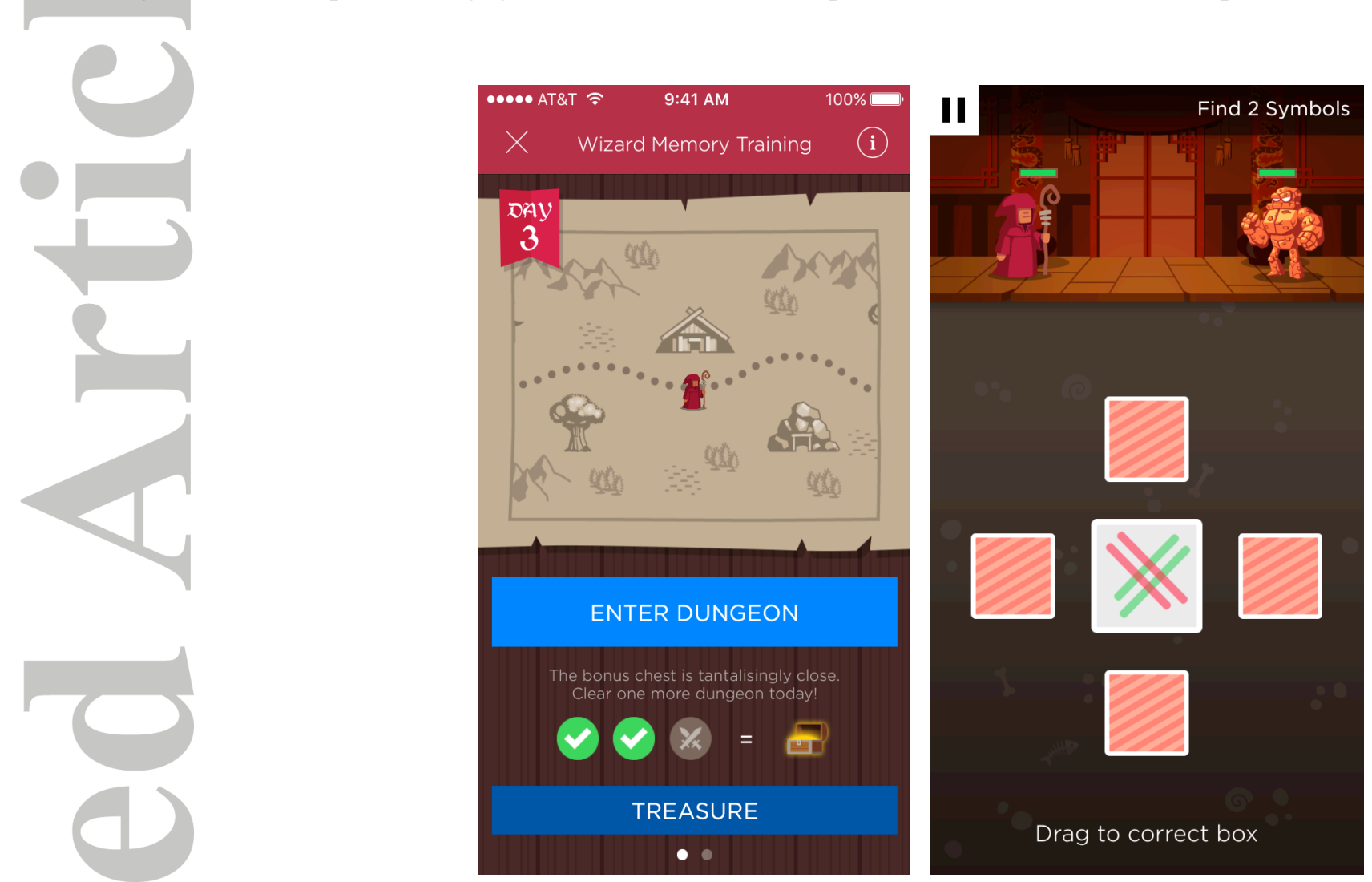

